BULL. AUSTRAL. MATH. SOC.

VOL. $2(1970), 81-88$.

\title{
On the extension of orders in ordered modules
}

\section{P. Ribenboim}

We introduce the notion of a positively independent set of elements in an ordered module. With this concept we determine a necessary and sufficient condition which insures that on a strictly ordered module over a strictly ordered ring there exists a strict total order refining the given order. This generalizes a previous result of Fuchs, concerning the case of ordered abelian groups.

As an application, let $R$ be a strictly ordered totally ordered ring and let $M$ be the $R$-module of all mappings from a set $I$ into $R$, with pointwise order; then this order on $M$ may be refined to a strict total order.

Let $R$ be a (commutative associative) ordered ring (with unit element $1 \neq 0)$, let $P_{R}=\{r \in R \mid r \geq 0\}$ be the cone of positive elements of $R$ (with respect to the given order). That is $P_{R}+P_{R} \subseteq P_{R}, P_{R} \cdot P_{R} \subseteq P_{R}, P_{R} \cap\left(-P_{R}\right)=\{0\}$. Moreover we shall assume that $1 \in P_{R}$. If $P_{R} \cup\left(-P_{R}\right)=R$ we say that $R$ is totally ordered.

We say that $\left(R, P_{R}\right)$ is strictly ordered when: $r, r^{\prime} \in P_{R}$, $r r^{\prime}=0$ implies $r=0$ or $r^{\prime}=0$. For example, if $R$ is an ordered integral domain then it is strictly ordered. However, the ring $Z^{I}$ of integral-valued functions on a set, with pointwise order, is not strictly

Received 23 August 1969. 
ordered (when $I$ has at least two elements).

Let $M$ be an $R$-module. A subset $C$ of $M$ is called a cone when it satisfies the following properties: $C+C \subseteq C, P_{R} \cdot C \subseteq C$. A cone $P_{M}$ such that $P_{M} \cap\left(-P_{M}\right)=\{0\}$ defines an order on $M$, making $M$ into an ordered R-module: $m \geq m^{\prime}$ whenever $m-m^{\prime} \in P_{M}$. If $P_{M} \cup\left(-P_{M}\right)=M$ we say that $M$ is totally ordered.

We say that $\left(M, P_{M}\right)$ is a strictly ordered module over $\left(R, P_{R}\right)$ when:

$$
r \in P_{R}, x \in P_{M}, \quad x x=0 \text { implies } r=0 \text { or } x=0 \text {. }
$$

Thus $\left(R, P_{R}\right)$ is a strictly ordered ring when it is a strictly ordered module over itself.

For example, if $R$ is a strictly ordered ring, if $M=R^{I}$ is the $R$-module of all functions from $I$ to $R$, with pointwise order, then $M$ is a strictly ordered module over $R$.

Let $\left(R, P_{R}\right)$ be an ordered ring, let $\left(M, P_{M}\right)$ be an ordered module over $\left(R, P_{R}\right)$.

We say that the set $\left\{x_{1}, \ldots, x_{n}\right\}$ of elements of $M$ is positively independent when the following holds:

$$
\text { if } r_{i} \in P_{R} \text { and } \sum_{i=1}^{n} r_{i} x_{i} \in P_{M} \text { then each } r_{i}=0 \text {. }
$$

For example if $x \in P_{M}$ then $\{x\}$ is not positively independent.

a) The following conditions are equivalent:

1) for every $x \& P_{M}$ the set $\{x\}$ is positively independent;

2) if $0 \neq r \in P_{R}$ and $r x \in P_{M}$ then $x \in P_{M}$.

The proof is immediate.

We shall now indicate a generalization of Theorem 1, p. 113 in [1]:

THEOREM. Let $\left(M, P_{M}\right)$ be a strictly ordered module over the 
strictly ordered ring $\left(R, R_{R}\right)$. The following statements are equivalent:

1) There exists a total order $T_{M}$ on $M$ such that $P_{M} \subseteq T_{M}$ and $\left(M, T_{M}\right)$ is a strictly ordered module over $\left(R, P_{R}\right)$;

2) if $a_{1}, \ldots, a_{n}$ are non-zero elements of $M$, there exist $\varepsilon_{1}, \ldots, \varepsilon_{n} \in\{1,-1\}$ such that the set $\left\{\varepsilon_{1} a_{1}, \ldots, \varepsilon_{n} a_{n}\right\}$ is positively independent in $\left(M, P_{M}\right)$.

Proof: $1 \rightarrow 2$. Let $a_{1}, \ldots, a_{n}$ be non-zero elements of $M$; then either $a_{i} \in T_{M}$ or $-a_{i} \in T_{M}$. Let $\varepsilon_{i} \in\{1,-1\}$ be such that $-\varepsilon_{i} \alpha_{i} \in T_{M}$. Then $\left\{\varepsilon_{1} a_{1}, \ldots, \varepsilon_{n} a_{n}\right\}$ is positively independent. For if $r_{i} \in P_{R}$ and $\sum_{i=1}^{n} r_{i} \varepsilon_{i} a_{i} \in P_{M} \subseteq T_{M}$, since $r_{i} \varepsilon_{i} a_{i} \in-T_{M}$ then $r_{i}\left(-\varepsilon_{i} a_{i}\right)=0$ for every $i=1, \ldots, n$. But $\left(M, T_{M}\right)$ is strictly ordered, hence $r_{i}=0$ for every $i=1, \ldots, n$.

$2 \rightarrow 1$. To prove this implication, we shall need a lemma. For every element $a \in M$ we denote by $C(a)$ the intersection of all cones of $M$ containing $a ; C(a)$ is clearly a cone, namely $C(a)=P_{R} a$.

LEMMA. Let $\left(M, P_{M}\right)$ be a strictly ordered module over $\left(R, P_{R}\right)$ satisfying condition (2). If $a \in M$ then either $P_{M}+C(a)$ or $P_{M}+C(-a)$ defines a strict order on $M$, satisfying condition (2).

Proof of the Lemma. The lemma is trivial when $a=0$, so we may suppose that $a \neq 0$. We assume that $M$ contains non-zero elements $a_{1}, \ldots, a_{n}$ and non-zero elements $b_{1}, \ldots, b_{m}$ such that for all $\varepsilon_{i}, n_{j} \in\{1,-1\}$ the sets $\left\{a, \varepsilon_{1} a_{1}, \ldots, \varepsilon_{n} a_{n}\right\},\left\{-a, \eta_{1} b_{1}, \ldots, \eta_{m} b_{m}\right\}$ are not positively independent.

Then for all $\delta, \varepsilon_{i}, \eta_{j} \in\{1,-1\}$ the sets $\left\{\delta a, \varepsilon_{1} a_{1}, \ldots, \varepsilon_{n} a_{n}, \eta_{1} b_{1}, \ldots, \eta_{m} b_{m}\right\}$ are not positively independent. This contradicts condition (2). 
Hence, there are two possibilities:

1) for all non-zero elements $a_{1}, \ldots, a_{n} \in M$ there exist $\varepsilon_{i} \in\{1,-1\}$ such that $\left\{a, \varepsilon_{1} a_{1}, \ldots, \varepsilon_{n} a_{n}\right\}$ are positively independent; in particular $\{a\}$ is positively independent and $P_{M} \cap C(a)=0$.

2) for all non-zero elements $a_{1}, \ldots, a_{n} \in M$ there exist $\varepsilon_{i} \in\{1,-1\}$ such that $\left\{-a, \varepsilon_{1} a_{1}, \ldots, \varepsilon_{n} a_{n}\right\}$ are positively independent; in particular $P_{M} \cap C(-\alpha)=0$.

$$
\begin{aligned}
& \text { In case (1) let } P_{M}^{\prime}=P_{M}+C(-a) \text {; } \\
& \text { in case (2) let } P_{M}^{\prime}=P_{M}+C(a) \text {. }
\end{aligned}
$$

Then clearly $P_{M}^{\prime}+P_{M}^{\prime} \subseteq P_{M}^{\prime}$ and $P_{R} P_{M}^{\prime} \subseteq P_{M}^{\prime}$. Now we show condition (2) for $P_{M}^{\prime}$ (for example in the first case). Let $a_{1}, \ldots, a_{n}$ be non-zero elements of $M$, let $\varepsilon_{i} \in\{1,-1\}$ be such that $\left\{a, \varepsilon_{1} a_{1}, \ldots, \varepsilon_{n} a_{n}\right\}$ are positively independent (relatively to $P_{M}$ ). We show that if $r_{i} \in P_{R}$ and $\sum_{1}^{n} r_{i} \varepsilon_{i} a_{i} \in P_{M}^{\prime}$ then $r_{i}=0, \forall i=1, \ldots, m$. For $\sum_{1}^{n} r_{i} \varepsilon_{i} a_{i}=x-r a$ with $x \in P_{M}, r \in P_{R}$; thus $r a+\sum r_{i} \varepsilon_{i} a_{i} \in P_{M}$, hence $r=r_{i}=0$, $\forall i=1, \ldots, n$.

From this follows $P_{M}^{\prime} \cap\left(-P_{M}^{\prime}\right)=0$. Because, if $0 \neq x \in P_{M}^{\prime} \cap\left(-P_{M}^{\prime}\right)$ then $x,-x \in P_{M}^{\prime}$, so the sets $\{x\},\{-x\}$ are not positively independent, against (2).

Hence $P_{M}^{\prime}$ defines an order on $M$ which makes it strictly ordered over $\left(R, P_{R}\right)$. In fact, let $0 \neq r \in P_{R}, x-s a \in P_{M}^{\prime}$, with $x \in P_{M}$, $s \in P_{R}$ and assume $r(x-s a)=0$, so $r x=r s a \in P_{M} \cap C(a)=0$; since the order $P_{M}$ is strict then $x=0 ;$ since $\{a\}$ is positively independent then $r s=0$; but $\left(R, P_{R}\right)$ is a strictly ordered ring, hence $s=0$; so $x-s a=0$.

Thus we have established the lema. 
Continuation of the proof of the Theorem. We consider all subsets $Q$ of $M$ satisfying

a) $P_{M} \subseteq Q$,

b) $Q+Q \subseteq Q$,

c) $P_{R^{Q}} \subseteq Q$,

d) $Q \cap(-Q)=0$,

e) if $r \in P_{R}, x \in Q$ and $r x=0$ then either $r=0$ or $x=0$,

f) condition (2) is satisfied by $Q$.

The family 2 of such subsets contains $P_{M}$. If $\left(Q_{i}\right)_{i=1,2, \ldots}$ is any strictly increasing chain of subsets in $Q$, let $Q=U_{l}^{\infty} Q_{i}$; then $Q \in Q$. Everything but $(f)$ is immediate. Now we check ( $f$ ). Let $a_{1}, \ldots, a_{n} \in M$; for every $i$ there exists $\varepsilon_{j}^{i} \in\{1,-1\} \quad(j=1, \ldots, n)$ such that $\varepsilon_{1}^{i} a_{1}, \ldots, \varepsilon_{n}^{i} a_{n}$ are positively independent (with respect to $\left.Q_{i}\right)$. Since there are only finitely many $n$-tuples of elements $1,-1$, then there exists an infinite chain $Q_{i_{1}} \subset Q_{i_{2}} \subset \ldots \subset Q_{i_{m}} \subset \ldots$ such $\left(\varepsilon_{1}^{i_{1}}, \ldots, \varepsilon_{n}^{i_{1}}\right)=\left(\begin{array}{c}i_{2} \\ \varepsilon_{1}\end{array}, \ldots, \varepsilon_{n}^{i_{2}}\right)=\ldots=\left(\begin{array}{c}i_{m} \\ \varepsilon_{1}\end{array}, \ldots, \varepsilon_{n}^{i_{m}}\right)=\ldots$. Let $\delta_{j}=\varepsilon_{j}^{i}$ for $m=1,2, \ldots, j=1, \ldots, n$.

Then $\delta_{1} a_{1}, \ldots, \delta_{n} a_{n}$ are positively independent over $Q$; for if $r_{j} \in P_{R}$ and $\sum_{j}^{n} r_{j} \delta_{j} a_{j} \in Q$ then there exists $m$ such that $\sum_{1}^{n} r_{j} \delta_{j} a_{j} \in Q_{i_{m}}$, hence $r_{j}=0$ for $j=1, \ldots, n$.

Thus $Q$ is inductive and by Zorn's Lemma, there exists a maximal element $T_{M} \in 2$.

Now, let $a \in M$. By the lemma, either $T_{M}+C(a)$ or $T_{M}+C(-a)$ defines an order satisfying condition (2) which is strict. By the 
maximality of $T_{M}$ we must have $a \in T_{M}$ or $-a \in T_{M}$, showing that $T_{M}$ is a total order on $M$.

We shall turn to the special case where $\left(R, P_{R}\right)$ is a strictly ordered ring, $M=R^{I}$ is the R-module of all functions from $I$ to $R$ and $P_{M}=\left\{f \in M \mid f(x) \in P_{R}\right.$ for every $\left.x \in I\right\}$.

Let us consider the following condition:

3) If $f_{1}, \ldots, f_{n}$ are non-zero elements of $M=R^{I}$ there exists $k, I \leq k \leq n$, elements $x_{1}, \ldots, x_{k} \in I$, a partition of $\{1, \ldots, n\}$ into disjoint non-empty subsets $S_{1}, \ldots, S_{k}$ and $\varepsilon_{1}, \ldots, \varepsilon_{n} \in\{-1,1\}$ such that

$$
\left\{\begin{aligned}
\varepsilon_{i} f_{i}\left(x_{j}\right) & <0 \text { when } i \in S_{j}, \\
f_{i}\left(x_{j}\right) & =0 \text { when } i \in S_{j+1} \cup \ldots \cup S_{k} .
\end{aligned}\right.
$$

We prove:

b) If $\left(R, P_{R}\right)$ is a strictly ordered ring then condition (3) implies condition (2) of the theorem.

Proof. Let $f_{1}, \ldots, f_{n}$ be non-zero elements of $M$. We choose $x_{1}, \ldots, x_{k}, s_{1}, \ldots, s_{k}$ and $\varepsilon_{1}, \ldots, \varepsilon_{n}$ as indicated in the hypothesis, and we proceed to show that if $r_{1}, \ldots, r_{n} \in P_{R}$ and $\sum_{i=1}^{n} r_{i} \varepsilon_{i} f_{i} \in P_{M}$ then each $r_{i}=0$. We have $\sum_{i=1}^{n} r_{i} \varepsilon_{i} f_{i}\left(x_{1}\right) \in P_{R}$, but $f_{i}\left(x_{1}\right)=0$ when $i \in S_{2} \cup \ldots \cup S_{k}$, hence $\sum_{i \in S_{1}} r_{i} \varepsilon_{i} f_{i}\left(x_{1}\right) \in P_{R}$. From $\varepsilon_{i} f_{i}\left(x_{1}\right)<0$ when $i \in S_{1}$ we deduce that $\sum_{i \in S_{1}} r_{i} \varepsilon_{i} f_{i}\left(x_{1}\right) \in P_{R} \cap\left(-P_{R}\right)=\{0\}$. Thus ${ }^{r_{i}} \varepsilon_{i} f_{i}\left(x_{1}\right)=0$ for every $i \in S_{1}$. Since $\left(P, R_{P}\right)$ is strictly ordered and $r_{i}\left(-\varepsilon_{i} f_{i}\left(x_{1}\right)\right)=0$ with $-\varepsilon_{i} f_{i}\left(x_{i}\right)>0$ we deduce that $r_{i}=0$ for $i \in S_{1}$. 
So we have $\sum_{i \xi S_{1}} r_{i} \varepsilon_{i} f_{i} \in P_{M}$ and we may proceed by induction showing successively that $r_{i}=0$ for every $i \in S_{j}$ and $j=1, \ldots, k$, hence that $r_{i}=0$ for every $i=1, \ldots, n$.

Now we prove:

c) If $\left(R, P_{R}\right)$ is a totally ordered ring then condition (3) is satisfied by $M=R^{I}$ with pointwise order.

Proof. Let $f_{1}, \ldots, f_{n}$ be non-zero elements of $M=R^{I}$, let $x_{1} \in I$ be such that $f_{1}\left(x_{1}\right) \neq 0$ and $s_{1}=\left\{i \mid 1 \leq i \leq n, f_{i}\left(x_{1}\right) \neq 0\right\}$. Since $\left(R, P_{R}\right)$ is totally ordered, for every $i \in S_{1}$ there exists $\varepsilon_{i} \in\{-1,1\}$ such that $\varepsilon_{i} f_{i}\left(x_{1}\right)<0$. If $S_{1}=\{1, \ldots, n\}$ then condition (3) is satisfied with $k=1$.

If $S_{1} \neq\{1, \ldots, n\}$ let $n_{2}$ be the smallest integer such that $n_{2} \notin s_{1}$ (thus $\left.1<n_{2} \leq n\right)$; since $f_{n_{2}} \neq 0$ and $f_{n_{2}}\left(x_{1}\right)=0$ there exists $x_{2} \in I, x_{2} \neq x_{1}$ such that $f_{n_{2}}\left(x_{2}\right) \neq 0$; let $S_{2}=\left\{i|i| S_{1}, f_{i}\left(x_{2}\right) \neq 0\right\}$. Since $\left(P, R_{P}\right)$ is totally ordered, for every $i \in S_{2}$ there exists $\varepsilon_{i} \in\{-1,1\}$ such that $\varepsilon_{i} f_{i}\left(x_{2}\right)<0$. If $S_{1} \cup S_{2} \neq\{1, \ldots, n\}$ we may proceed in this way, and after a finite number of steps we establish the validity of condition (3).

We have therefore shown:

d) Let $\left(R, P_{R}\right)$ be a strictly ordered, totally ordered ring; let $M=R^{I}$ be the ordered R-module with pointwise order. Then there exists a total order $T_{M}$ on $M$ such that $P_{M} \subseteq T_{M}$ and $\left(M, T_{M}\right)$ is a strictly ordered module over $\left(R, P_{R}\right)$. 


\section{References}

[1] L. Fuchs, Partially ordered algebraic systems, (Pergamon Press, Oxford, London, New York, Paris, 1963).

[2] P. Ribenboim, "On ordered modules", J. Reine Angew. Math. 225 (1967), 220-146.

Queen's University,

Kingston, Ontario. 